\title{
A Comparison of the Cingulum Tract in ALS-B Patients and Controls Using Kernel Matching
}

\author{
Sander van Noorden ${ }^{1}$, Matthan Caan ${ }^{1,2}$, Maaike van der Graaff ${ }^{2}$, \\ Lucas van Vliet ${ }^{1}$, and Frans $\operatorname{Vos}^{1,2}$ \\ ${ }^{1}$ Quantitative Imaging Group, Delft University of Technology, NL \\ ${ }^{2}$ Academic Medical Center, University of Amsterdam, NL \\ m.w.a.caan@amc.uva.nl
}

\begin{abstract}
Amyotrophic lateral sclerosis (ALS) is a progressive motor neuron disease with poor prognosis. Previous DW-MRI based studies in ALS on WM tracts showed a decrease of FA in tracts related to the motor system. Recent evidence suggests that extra-motor tracts are also affected by ALS. This paper aims to analyse the cingulum tracts of ALS patients and controls. To do so, we introduce kernel matching, a novel method to obtain optimal correspondence between the white matter tracts. The orientation of tract tensors in atlas space as well as the global tract shape are employed as prior information. The method proved successful to reduce the large variance of tensor shape features along the cinguli emanating from registration errors. Only after applying the proposed kernel matching method we found a significant increase in the tensor norm of both cinguli. We hypothesize that the degeneration of fibers increases tensor norm.
\end{abstract}

\section{Introduction}

Amyotrophic lateral sclerosis (ALS) is a progressive motor neuron disease featuring upper as well as lower motor neuron degeneration and poor prognosis. The exact cause of ALS is currently unknown.

Diffusion weighted MRI (DW-MRI) is a powerful modality to study the degeneration of white matter (WM) tracts. Previous DW-MRI based studies on ALS performed either voxel-based analysis (VBA) of WM tracts after a fulltensor non-rigid registration [1] or tract-based spatial statistics (TBSS) after a fractional anisotropy (FA)-based registration [2]. Both studies showed a decrease of FA in tracts related to the motor system such as the corticospinal tract (CST). Sage [1] reported an increase of the mean diffusivity along the CST.

Recent evidence suggests that extra-motor tracts are also effected by ALS. For instance, both 1 and 2] found a lower FA in the body of the corpus callosum (CC). Related fMRI studies showed a decrease of blood oxygen level-dependent (BOLD) contrast in the medial part of the cingulum during the execution of motor tasks 3. So far the cingulum has not been reported to be structurally involved in ALS. 
The analysis of atlas-tract statistics, such as in [4, relies on a good registration. Particularly, the assessment of smaller tracts is rather sensitive to registration errors, which increase the variance in the tensor shape features. These registration errors result from spatial regularization and ignore small local mismatches in order to find a transformation that is continuous at a larger scale [5]. TBSS provides a method that is less sensitive to registration errors as it projects tensor features on the closest location of the FA skeleton. While doing so, differences in orientation between the projected tensor and the target on the FA skeleton are ignored. Hence, this may result in a misregistration for closely spaced tracts such as the cingulum and the CC. Alternatively, fibers may be tracked in subject space, transformed to the atlas space followed by clustering 6]. Although more accurate, this method is less precise due to the low SNR of the subjects' datasets in which tracking is performed.

This paper aims to analyse the cingulum tracts of ALS patients and controls. Our study employs a novel kernel matching approach that is used for registration of the cingulum tracts. The kernel matching locally improves tract correspondence by employing the orientation of tract tensors in atlas space as well as the global tract shape as prior information. By doing so a projection step such as in TBSS is rendered superfluous. Statistics are computed in subject space to avoid unnecessary interpolation of the tensor data.

\section{Method}

\subsection{Tract Segmentation in a Population-Specific Atlas}

We consider tract segmentation in a population-specific tensor atlas as an initialization for our kernel matching method. This atlas contains the complete diffusion information [7] and is built for this study using a non-rigid viscous fluid registration model [5]. Fiber tractography is performed in the atlas using the FACT algorithm [8]. The resulting fiber points are clustered and a spline is fitted through the cluster center points $\mathbf{c}_{\text {atlas }}$, resulting in a smooth centerline 9]. Tracts are represented by this set of cluster centers points, the original fiber points and the cluster-membership of each fiber point to a cluster center defined in [9] as the Gaussian-weighted distance ( $\sigma=3 \mathrm{~mm}$, approx. 1 voxel). The inverse of each subject-to-atlas transformation is computed, which effectively transforms atlas tracts back to subject space.

\subsection{Optimize Cluster Location in Subject Space by Kernel Matching}

Kernel matching aims at improving the atlas tract to subject transformation. The atlas tract may be warped in the normal plane $\tau\left(\mathbf{c}_{\text {atlas }}\right)$ to its local orientation, to achieve correspondence with the local subject tensor orientation. From the tensors, only orientation information is employed. Given a principal eigenvector $\mathbf{v}_{\mathbf{i}}=\left(v_{x}, v_{y}, v_{z}\right)^{T}$, an orientation mapping can be defined by considering the outer-product $\mathbf{M}_{\mathbf{i}}=\mathbf{v}_{\mathbf{i}} \mathbf{v}_{\mathbf{i}}^{\mathbf{T}}$. The resulting 9 -tuple can be reduced to a 5 -dimensional normalized vector $\mathbf{w}_{\mathbf{i}}[10$ : 


$$
\mathbf{w}_{\mathbf{i}}=\sqrt{\frac{3}{4}}\left(v_{x}^{2}-v_{y}^{2}, 2 v_{x} v_{y}, 2 v_{x} v_{z}, 2 v_{y} v_{z}, \frac{1}{\sqrt{3}}\left(2 v_{z}^{2}-v_{x}^{2}-v_{y}^{2}\right)\right) .
$$

This operation ensures that antipodal vectors are mapped to the same point in the 5-dimensional space. We define the similarity $0 \leq s \leq 1$ in orientation between two diffusion tensors as $s\left(\mathbf{w}_{1}, \mathbf{w}_{2}\right)=\frac{1}{2}\left(\left(\mathbf{w}_{1} \cdot \mathbf{w}_{2}\right)+1\right)$. The location of an atlas cluster center point is updated by adjusting $\mathbf{q} \in \tau\left(\mathbf{c}_{\text {atlas }}\right)$ such that a matching kernel $\eta$ is found in a subject that maximizes kernel similarity, $\arg \max _{\mathrm{q}} \mathrm{S}(\mathbf{q})$. $S(\mathbf{q})$ is defined as:

$$
S(\mathbf{q})=\left\{\sum_{p \in \eta} s\left(\mathbf{w}_{\mathbf{p}, \text { atlas }}, \mathbf{w}_{\mathbf{p}+\mathbf{q}, \text { subject }}\right) \cdot s\left(\mathbf{w}_{\mathbf{p}, \text { atlas }}, \mathbf{w}_{\mathbf{c}_{\text {atlas }}}\right)\right\} .
$$

In this equation, the first term decribes the similarity in orientation between atlas and subject. The second term ensures that adjacent bundles with a different orientation are ignored.

The cubic kernel $\eta$ gives an equal weight to all elements. $\eta$ is sized $5 \times 5 \times 5$ voxels $\left(1.0 \times 1.0 \times 1.1 \mathrm{~cm}^{3}\right)$, which is slightly broader than the average width of the small WM tracts which we are interested in. We limit $\eta$ to WM voxels that have $\mathrm{FA}>0.2$ and mode $>0$ (see below) such that their principal eigenvector is well-defined.

Contrast in WM tract orientation is largest in the direction perpendicular to the tract. Furthermore, the registration error of the atlas tract is expected to be smaller than $1.5 \mathrm{~cm}$. Therefore, we update $\mathbf{c}_{\text {atlas }}$ in the plane $\tau\left(\mathbf{c}_{\text {atlas }}\right)$ normal to the local tract orientation. $\tau\left(\mathbf{c}_{\text {atlas }}\right)$ is of small size $1.5 \times 1.5 \mathrm{~cm}^{2}$, discretized at a finer 0.5 voxel-size interval resulting in $17 \times 17$ points.

Interpolation of tensor values is done in a Log-Euclidean space to prevent tensor swelling effects [11. The atlas tensor field is transformed to subject space before $S(\mathbf{q})$ is evaluated and tensors are rotated accordingly.

Cluster locations in subject space are updated to the position $\mathbf{c}_{\text {atlas }}+\mathbf{q}$ where $S(\mathbf{q})$ is maximal. The resulting centerline through these updated cluster locations is regularized by fitting a $b$-spline followed by projecting each cluster to the closest point on the spline. One iteration of the algorithm was performed, as more repetitions did not alter the solution. The location of fiber points is updated by interpolation of the cluster translations using the cluster-membership 9] as weights.

\subsection{Tensor Shape and Tract Statistics}

Conventionally, DW-MRI analyses of WM tracts rely on the FA to describe the tensor shape. Recently, it was shown that the tensor shape is entirely described by three features: FA, mode and norm, which form an orthogonal set of features 12. FA measures the anisotropy, mode the type of anisotropy (ranging from -1 for planar to +1 for linear anisotropy) and norm the amount of diffusivity.

These features are calculated at cluster centers as a weighted sum of the feature at all fiber points employing the cluster-membership as weights. Patient 
and control feature profiles are compared per cluster center using a $t$-test with a modest significance threshold of $p<0.05$ (uncorrected), because of a small population size.

\section{Results}

\subsection{Data Acquisition}

Over a period of 3 years we consecutively included all 11 patients that entered four neuromuscular outpatient clinics in the Netherlands (University Medical Centres of Amsterdam, Utrecht, Rotterdam, and the Catharina Hospital in Eindhoven) and met our inclusion criteria (ALS, bulbar onset of disease) as well as 11 age matched controls. DTI data were acquired on a 3T scanner (Intera, Philips Healthcare, Best, The Netherlands). The spatial resolution was $2.0 \times 2.0 \times 2.2$ $\mathrm{mm}$, per patient 64 axial slices of matrix size 128x128 were acquired for 32 gradient directions with a diffusion weighting of $b=1000 \mathrm{smm}^{-2}$. Additionally, one set of images was acquired without any diffusion-weighting. Eddy current distortions were corrected for by an affine registration in the phase direction [13.

\subsection{Tract-Based Spatial Statistics}

TBSS was performed after tensor-based registration (section 2.1). Results are shown in fig. 1. A decreased FA in the corticospinal tract (CST) and in the body of the corpus callosum (CC) is observed. The norm is increased in a few
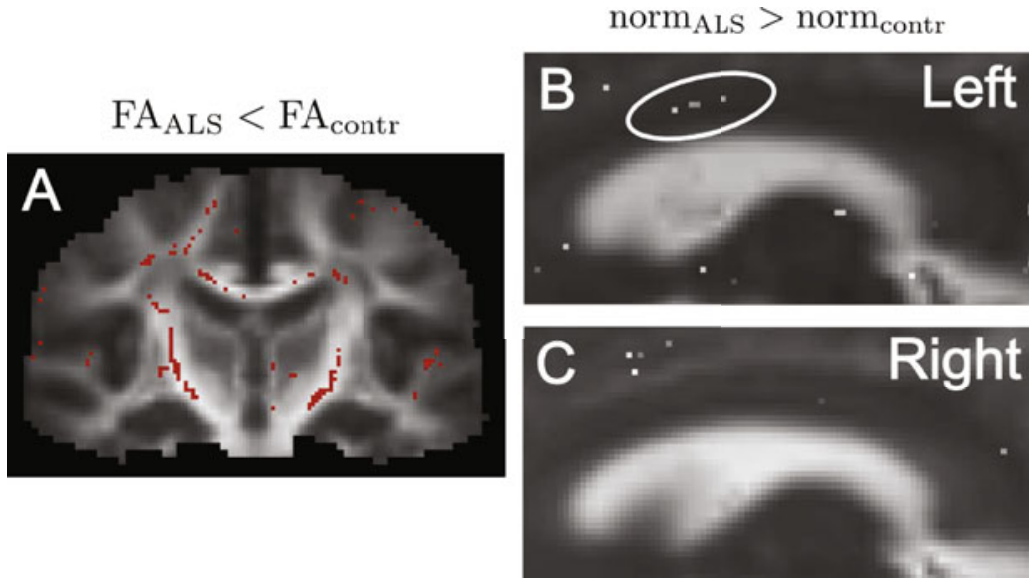

Fig. 1. TBSS results on FA (a) and norm of the left (b) and right (c) cingulum. Marked voxels show a significant decrease in FA (a) or a significant increase in norm $(b, c)$ between ALS patients and controls according to a t-test with $p<0.05$ (uncorrected). (a) Shows the FA is lowered for the CST and CC. (b) Shows a small effect for the norm of the left cingulum in the annotated area. (c) No significant effect is observed in the right cingulum. 

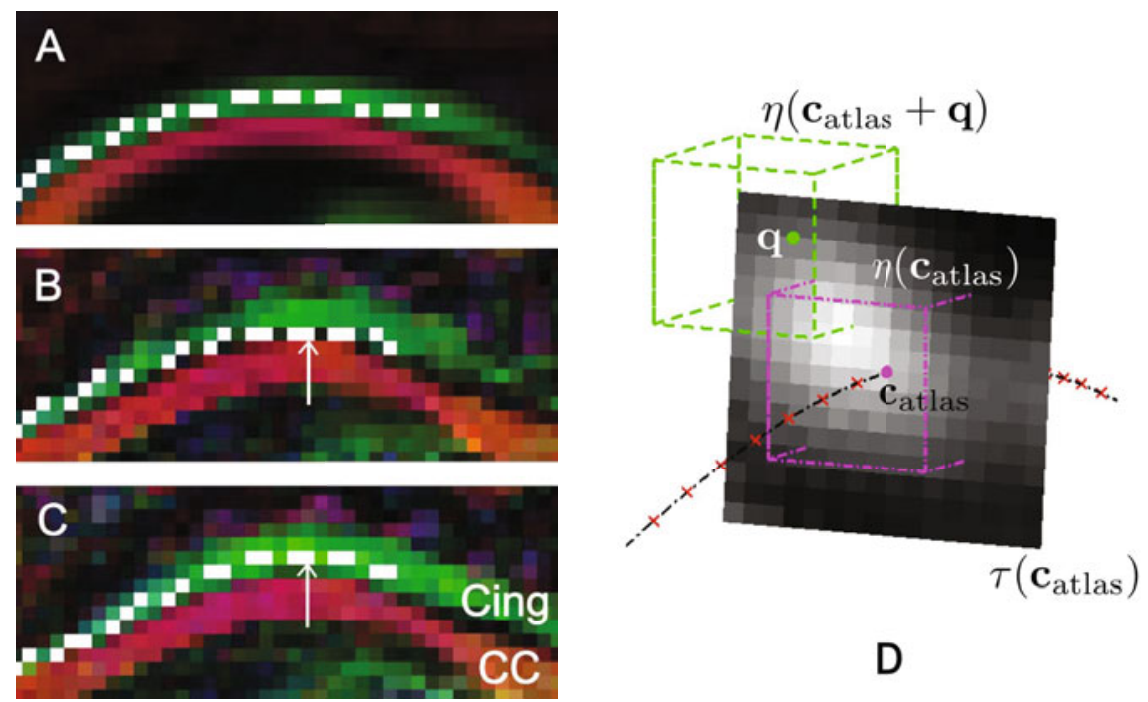

D

Fig. 2. Location of right cingulum cluster centers in atlas (a) and in subject before (b) and after (c) kernel matching. (d) Kernel similarity $S(\mathbf{q})$ evaluated for $\tau\left(\mathbf{c}_{\text {atlas}}\right)$ around marked cluster in (b), with the kernel $\eta\left(\mathbf{c}_{\text {atlas }}+\mathbf{q}\right)$ displayed at an arbitrary location.

voxels of the left cingulum, whereas no differences could be found in the right cingulum. According to the authors, TBSS is less suited for this particular area as the projection direction towards a skeleton with a tubular rather than a sheet-like topology, such as the inferior part of the cingulum, is not uniquely defined. In these areas the TBSS method employs a local search for maximum FA within a circular space in the appropriate axial slice, rather than along a single perpendicular direction [14.

\subsection{Kernel Matching}

Both cingulum tracts were tracked in the atlas and a centerline through its cluster center points was derived. The atlas cluster center points, depicted in fig. 2a, were transformed to subject space. Fig. 20 illustrates that in a representative subject these cluster center points did not align with the cingulum but instead were incorrectly positioned in between the cingulum and CC, due to a small registration error.

Subsequently, kernel matching was applied to obtain better correspondence between the atlas tracts and the subjects' tensor orientation field. Figure 2 $\mathrm{d}$ shows the kernel similarity $S(\mathbf{q})$ evaluated in the normal plane $\tau\left(\mathbf{c}_{\text {atlas }}\right)$ of the annotated cluster center point in fig. 2 b. A high contrast in $S(\mathbf{q})$ may be observed for a small region adjacent to the initialized cluster center point $\mathbf{c}_{\text {atlas }}$. This region corresponds to the cross section of the subjects' cingulum bundle at that particular location. The initial cluster center point (purple) is clearly 


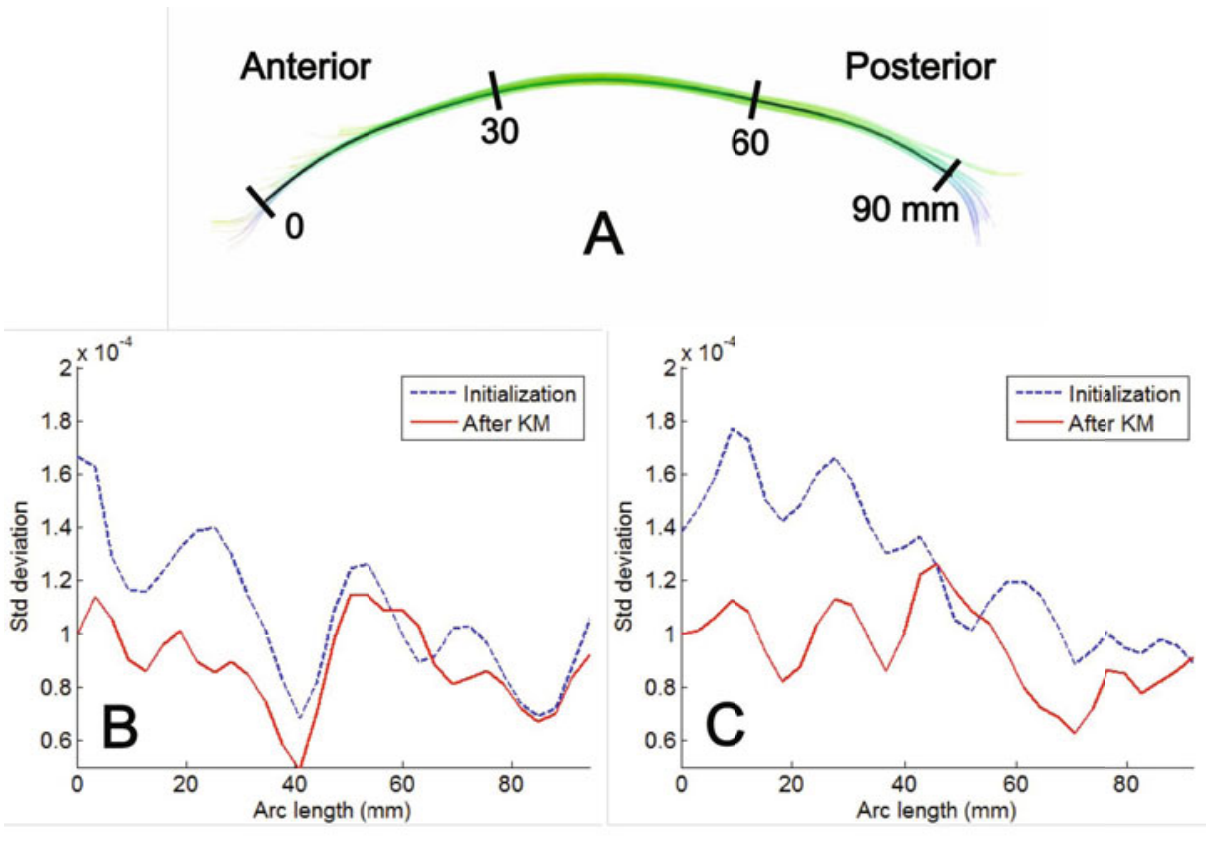

\section{Left cingulum}

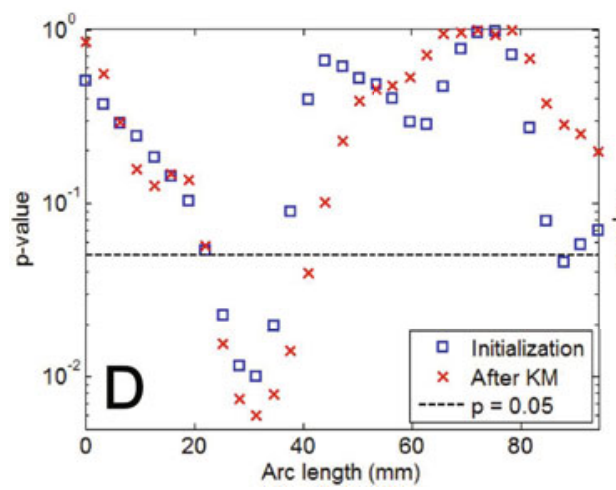

Right cingulum

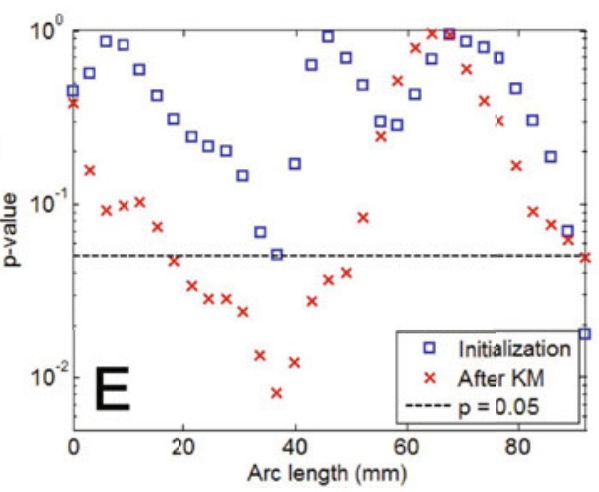

Fig. 3. (a) Defining arc length to parameterize the right cingulum in atlas space. (b,c) Standard deviation of the norm for the left (b) and right (c) cingulum in controls before and after kernel matching. Mean norm for both cinguli is approximately $1.5 \times 10^{-3}$ $(\mathrm{d}, \mathrm{e}) \mathrm{T}$-test results for the difference in norm between patients and controls evaluated at the left (d) and right (e) cingulum cluster centers. The dashed line denotes the significance threshold $(p<0.05)$. 
suboptimally positioned and will be translated by $\mathbf{q}$ to the voxel with maximal $S(\mathbf{q})$. Kernel matching results in a clear improvement in correspondence between the atlas tracts and this subjects' tensor orientation field (see fig. 2.

Tensor feature profiles of tracts evaluated after kernel matching are expected to have a lower standard deviation since these tracts are better aligned to the subject data. This reduction is demonstrated in the norm profiles of both cinguli of the control group in fig. 3b-c. The standard deviation is reduced up to $30 \%$ for clusters in the anterior and medial parts of the cingulum. Similar reductions are observed for the FA and mode profiles (data not shown).

Feature profiles that are evaluated before the application of kernel matching show an increase of tensor norm only in the left cingulum that is similar to the TBSS result, see fig. 3 $3 \mathrm{~d}$. FA and mode profiles do not show an effect (data not shown). The reduced standard deviation after kernel matching enables a more accurate comparison of patient and control profiles. This results in an enlarged significance of the effects observed in the left cingulum and in addition a similar effect in the right cingulum, see fig. [3 d-e. A permutation test as in [1] without correction for multiple comparisons gives exactly the same results as the paired t-test. The suprathreshold multiple comparison correction [15] indicates a significant difference of the entire right $\mathrm{CG}$, but not the left.

KM was also applied to other WM tracts of interest (CST, Inferior Longitudinal Fasciculus, Arcuate Fasciculus and Fornix) resulting in similar decreases in standard deviation as in the CG tracts. No additional significant differences between controls and patients were found.

\section{Discussion}

Both the atlas-tract based method without kernel matching and the TBSS analysis showed a difference in norm for the left cingulum. Only after applying the proposed kernel matching method we found a significant increase in the tensor norm of the right cingulum. This effect may be attributed to a higher initial variance, reduced after kernel matching. We hypothesize that the degeneration of fibers increases tensor norm. A related increase in tensor FA is also found but not significant, which may be due to the low number of included patients.

Future work might extend the application of our method to improve the correspondence of more sheet-like tracts by applying kernel matching to the skeleton points defined in the continuous medial representation suggested by [16]. Here, the matching may be restricted to translate cluster center points along the normal line to the sheet structure, instead of the normal plane of the fiber bundle.

\section{References}

1. Sage, C.A., Van Hecke, W., Peeters, R., Sijbers, J., Robberecht, W., Parizel, P., Marchal, G., Leemans, A., Sunaert, S.: Quantitative diffusion tensor imaging in amyotrophic lateral sclerosis: Revisited. Hum. Brain Mapp. 30(11), 3657-3675 (2009) 
2. Ciccarelli, O., Behrens, T.E., Johansen-Berg, H., Talbot, K., Orrell, R.W., Howard, R.S., Nunes, R.G., Miller, D.H., Matthews, P.M., Thompson, A.J., Smith, S.M.: Investigation of white matter pathology in als and pls using tract-based spatial statistics. Hum. Brain Mapp. 30(2), 615-624 (2009)

3. Lule, D., Diekmann, V., Kassubek, J., Kurt, A., Birbaumer, N., Ludolph, A.C., Kraft, E.: Cortical plasticity in amyotrophic lateral sclerosis: Motor imagery and function. Neurorehab. Neural. Re. 21(6), 518-526 (2007)

4. Goodlett, C., Fletcher, P., Gilmore, J., Gerig, G.: Group analysis of dti fiber tract statistics with application to neurodevelopment. Neuroimage 45, S133-S142 (2009)

5. Van Hecke, W., Leemans, A., D'Agostino, E., De Backer, S., Vandervliet, E., Parizel, P.M., Sijbers, J.: Nonrigid coregistration of diffusion tensor images using a viscous fluid model and mutual information. IEEE T. Med. Imaging 26(11), 1598-1612 (2007)

6. O'Donnell, L.J., Westin, C.F., Golby, A.J.: Tract-based morphometry for white matter group analysis. Neuroimage 45(3), 832-844 (2009)

7. Van Hecke, W., Sijbers, J., D'Agostino, E., Maes, F., De Backer, S., Vandervliet, E., Parizel, P.M., Leemans, A.: On the construction of an inter-subject diffusion tensor magnetic resonance atlas of the healthy human brain. Neuroimage 43(1), 69-80 (2008)

8. Jiang, H.Y., van Zijl, P.C.M., Kim, J., Pearlson, G.D., Mori, S.: Dtistudio: Resource program for diffusion tensor computation and fiber bundle tracking. Comput. Meth. Prog. Bio 81(2), 106-116 (2006)

9. Caan, M., Van Vliet, L., Majoie, C., Aukema, E., Grimbergen, K., Vos, F.: Spatial consistency in $3 \mathrm{~d}$ tract-based clustering statistics. In: Metaxas, D., Axel, L., Fichtinger, G., Székely, G. (eds.) MICCAI 2008, Part I. LNCS, vol. 5241, pp. 535542. Springer, Heidelberg (2008)

10. Rieger, B., Van Vliet, L.: A systematic approach to nd orientation representation. Image Vision Comput. 22, 453-459 (2004)

11. Arsigny, V., Fillard, P., Pennec, X., Ayache, N.: Log-euclidean metrics for fast and simple calculus on diffusion tensors. Magn. Reson. Med. 56(2), 411-421 (2006)

12. Kindlmann, G., Ennis, D.B., Whitaker, R.T., Westin, C.F.: Diffusion tensor analysis with invariant gradients and rotation tangents. IEEE T. Med. Imaging 26(11), 1483-1499 (2007)

13. Mangin, J., Poupon, C., Clark, C., Bihan, D., Bloch, I.: Eddy-current distortion correction and robust tensor estimation for $\mathrm{mr}$ diffusion imaging. In: Niessen, W.J., Viergever, M.A. (eds.) MICCAI 2001. LNCS, vol. 2208, pp. 186-193. Springer, Heidelberg (2001)

14. Smith, S.M., Jenkinson, M., Johansen-Berg, H., Rueckert, D., Nichols, T.E., Mackay, C.E., Watkins, K.E., Ciccarelli, O., Cader, M.Z., Matthews, P.M., Behrens, T.E.J.: Tract-based spatial statistics: Voxelwise analysis of multi-subject diffusion data. Neuroimage 31(4), 1487-1505 (2006)

15. Nichols, T.E., Holmes, A.P.: Nonparametric permutation tests for functional neuroimaging: A primer with examples. Hum. Brain Mapp. 15(1), 1-25 (2002)

16. Yushkevich, P.A., Zhang, H., Simon, T.J., Gee, J.C.: Structure-specific statistical mapping of white matter tracts. Neuroimage 41(2), 448-461 (2008) 